\title{
Hacking Jeff Minter's Virtual Light Machine: Unpacking the code and community behind an early software-based music visualizer
}

Eamonn Bell

Accepted for publication in Volume!

\section{Table of Contents}

Keywords/mots clés (English)

- counterculture / resistance

- digitality

- $\quad$ representation (visual)

- $\quad$ sound system

- technologies / devices

- $\quad$ websites / blogs / forums

- $\quad$ [hacking] (not in taxonomy)

- [music visualization] (not in taxonomy)

\section{Geographical index}

N/A

\section{Chrono index}

1990-2000 


\section{Author's biography}

Eamonn Bell holds a doctorate in music theory from Columbia University (2019), where he wrote a dissertation on the early history of computing in the analysis of musical scores, under the supervision of Joseph Dubiel. At Columbia, he designed and taught a course on the critique of digital music (2018), and instructed the undergraduate sections in history of Western music for nonmusicians (2018) and the fundamentals of music theory (2017). His research interests include: the history of technology as it relates to musical production and consumption in the twentieth century, with a focus on the first digital computers; the applied use of mathematical and contemporary computational techniques to solve problems in musicology and music theory; and visualizations of musical data. He also holds a bachelor's degree in Music and Mathematics from Trinity College, Dublin (TCD) (2013). He recently begain a two-year research fellowship at his alma mater, where he is working on the cultural history of optical sound-recording media.

\section{Resumé (Abstract)}

Foreshadowing in purpose and execution the music visualizers that were widely distributed with software media players during the early 2000s, Jeff Minter's Virtual Light Machine (VLM) was distributed in the firmware of the commercially unsuccessful Atari Jaguar CD games console, which was released in 1995. The VLM was designed to play an audio CD and generate real-time animations in more-or-less tightly coupled synchrony with music. The following year, Minter published "YaK's Quick Intro to VLM Hacking”, an online guide describing how to customize the visualizer's 81 graphical presets that revealed a hidden menu in the software. Minter's software work, not widely known outside of the community of video game historians and enthusiasts, deserves inclusion in a broader history of consumer music visualization technology. I draw on borndigital primary sources-including newsgroup posts, web pages, and the original code for the VLM itself-to understand the extent to which the practices explicitly and implicitly endorsed by Minter are congruent with our contemporary understanding of hacking. 


\section{Introducing the Virtual Light Machine}

On June 23, 1994, a breathless newswire from the Atari Corporation announced the impending release of its Jaguar CD console add-on accessory for the US market. Indulging in a number of dubious mixed metaphors, the press release described one of the accessory's more distinctive selling points: it was to be distributed with a built-in, interactive music visualizer, designed and programmed by the British video game artist Jeff Minter.

Thanks to the many talents of Jeff Minter, author of the award-winning Tempest $2000^{\mathrm{TM}}$, Atari's new CD-ROM includes the Virtual Light Machine ${ }^{\mathrm{TM}}$ (VLM). No other game system has VLM. VLM literally looks at audio compact discs as they are played and spontaneously produces a spectrum analysis of every incoming sound. This information is assembled in a frequency map of the music and assigned complex visual equivalents. Advanced 64-bit data processing makes it possible for the translation to take place almost faster than the ear can hear it so images are in perfect tune with the sound as it is played. The result is a stunning light show. VLM is perfect for parties and every other listening environment where audio compact discs are enjoyed (NEWSWIRE, 1994).

Promotional copy on the Jaguar CD's packaging (Figure 1) proclaimed that "VLM brings your music to life", and that owners could "pop in a music CD, sit back and watch as 65,000 colors warp, contort and distort to every beat, octave and power chord". Owners of the Virtual Light Machine (VLM) listened to the music of their choice as multicolored, pixillated graphics moved onscreen and appeared to change in response to the music heard.

[FIGURE 101_vlmboxhash.jpg]

Despite its attractive graphics processing power, the ill-fated Jaguar caused significant financial problems for Atari-they sold just 125,000 units world-wide between its release in 1993 and 1995, and were burdened with another 100,000 units that remained on inventory by the end of that year (ATARI CORPORATION, 1996). The Jaguar CD add-on (shown in Figure 2) for which Minter was commissioned to write the VLM was itself an even less widely available piece of consumer electronics.$^{1}$ Uncertainties about the precise extent of its failure, the commercial reach and impact of the Jaguar CD paled in comparison with that of its direct competitor: released on the last day of 1994, the CD-based Sony

\footnotetext{
${ }^{1}$ It is widely asserted that Atari sold only the first production run consisting 20,000 units of the Jaguar CD; there is some evidence from serial numbers and manufuacturing dates shared online by Jaguar CD owners that this is unlikely to be the case. For example, at https://atariage.com/forums/topic/156319-jaguarcd-real-production-quantity-and-hardware-variations.
} 
Playstation sold over 102 million units by March 2007 (SONY COMPUTER ENTERTAINMENT, 2011).

[FIGURE 2 02_jagcdandcontroller.jpg

The main interface offers conventional CD playback features; the media transport buttons (play, pause, skip forward, etc.) are displayed prominently. Like the majority of audioreactive software visualizers, the VLM is driven by continuous acoustic signals (i.e. the sounds themselves) and not discrete data that might be implied by an instrumental performance or derived from a musical score. ${ }^{2}$ When the user presses the up or down arrows on the D-pad, a overlay of a volume slider appears on the screen, allowing control of the playback volume, or "gain". If the gain is set too low, the visualizer's shapes remain sluggish and inactive, even if the track is uptempo; too high and the animation seems to respond hyperactively to the music. The finicky VLM does not always produce desirable audiovisual correspondences between, say, the pulsing bassline of an acid house track and the vibrating shapes on screen. Tweaking the playback gain appeared, for most users, to be the only way to adjust the visualizer's behavior.

[FIGURE 3 03_montage.jpg]

The VLM allowed players to select from an array of different types of visualizations. Minter used language already in common use in the audio synthesis world to describe how these different visualization strategies were arrayed: each distinct kind of visualization is called a "preset"; presets are organized into nine "banks", containing nine presets each, for a total of 81 preset visualization styles (Figure 3). Players used the idiosyncratic telephonestyle number pad on the Jaguar's controller to literally dial in the desired preset from a finite palette of options. Presets in the same bank share a basic visualization conceit with each family member constituting a variation on some underlying visual theme.

Little would the average player know Minter had hidden in VLM software the facility to create brand new presets by manipulating dozens of audio-visual software parameters opening up millions of new possibilities for visualization. Before I explain this hidden menu in greater detail, I begin by situating the VLM in the context of other similar devices that preceded it Then, I consider the specific senses in which "hacking" can be understood in relation to the device.

${ }^{2}$ cf. The Music Animation Machine (1985-) of Stephen Malinowski, which primarily consumes a symbolic representation of the score. BAIN (2008), 29-31. 
Finally, I discuss the advantages of studying this digital artifact through the close consideration of its code and of the online trace of its community of owners.

\section{Points of reference}

\section{Color organs, video synthesizers, music visualizers, lightsynths; oh my!}

Jeff Minter's VLM is part of a long line of devices that co-ordinate music and light in time: from the notorious ocular clavichord of Louis Bertrand Castel (1725) to the tastiera per luce that accompanied Scriabin's Prometheus (1915) and early-twentieth-century "color organs" in their image. The dawn of film spurred the Soviet experiments with draw-on sound, as well as those of Rudolf Pfenninger and Oskar Fischinger, the later also widely known for his experiments in "visual music" using cut-outs and other novel techniques for stop-motion animation. ${ }^{3}$ The mid-century witnessed Gordon Pask's cybernetic Musicolour (1953-1957) and the pieces of John and James Whitney (starting in the late 1950s), leading to the computer-controlled animations of Laurie Spiegel (1974-1976) and Lillian F. Schwartz (1970-). ${ }^{4}$ The desire to "see sound" is perhaps most recently attested to by the visualization software that was bundled with widely used audio player software including Nullsoft's Winamp (1997), Windows Media Player 7 (2000), and the Apple iTunes player (2001). Importantly, these audiovisual experiences were fully commodified, and widely accessible to the millions of PC owners. Unlike the preponderance of one-off or experimental devices represented by the selective, and like the VLM, this software brought real-time, reactive music visualization into the home.

${ }^{3}$ On the Soviet and Austro-German histories of drawn-on sound respectively, see: IzVOLOV (2014); LeVIN (2003). See also, PATTESON (2016), 106-113

${ }^{4}$ On the Whitney brothers' work, see PATTERSON (2009). On Gordon Pask's Musicolour, see PICKERING (2010), 313-321. For Spiegel on her graphic practice in her own words, see SPIEGEL (1998). A large selection of Schwartz's film work is available online at http://lillian.com/films/.

${ }^{5}$ Visualizer plugins were available for Winamp at least as early as 1998; the first version of the influential Advanced Visualization Studio (AVS) appeared with Winamp 2.61 (2000). 
A self-avowedly abbreviated account of this lineage has been sketched by Amy Alexander and Nick Collins; they acknowledge the futility of trying to "tie up all the historical threads of audiovisual performance" and focus primarily on contemporary VJ and live cinema work. ${ }^{6}$ Ideally complementary to those histories of abstract cinema provided by film studies, a comprehensive history of music and light with an explicitly musicological tint has yet to be completed. ${ }^{7}$ Karen Collins, William Gibbons, and Roger Moseley have each separately noted the intersection between video games and the development of new music visualizations, citing dozens of music-related video games that feature music visualization of some kind. Their examples range from simple rhythm games to fully-featured simulacra for musical performance, represented by Harmonix's Guitar Hero family of video games. ${ }^{8}$ Their ludomusicological work justifies a focus on games in the context of the history of music visualization, bringing insights from a medium ordinarily excluded from organological or film-history perspectives on the topic.

There are, nevertheless, notable points of contact between Minter's digital practice and avant-garde visual art in the twentieth century arising from the experimental use of video technology. For instance, Peter Sachs Collopy has traced the twentieth-century use of analog circuits to generate and modify video signals, through to the hybrid digital-analog systems that allowed for finegrained computer control of electronic circuitry (CoLlOPY, 2014). Collopy, like many of the artists whose work he cites, uses the umbrella term "video synthesizer" to refer to the various systems he discusses; the implied analogy is justified, since "designers modeled video synthesizers on audio synthesizers" (COLlOPY, 2014 : 74). Minter would have been sympathetic to this analogy, since uses the term "lightsynth" to refer not only his VLM, but also to its several predecessors, each of which share the aim of creating a more-or-less independent play of light. Here, I prefer the term "music visualizer" to describe such a device that, using the representation of a recording as an audio signal, produces animated images intended to be viewed in synchrony with musicwhether or not they require or facilitate interaction by their users. ${ }^{9}$

${ }^{6}$ AleXANDER and Collins (2017)

${ }^{7}$ Though see WHYTE (2019) for work in precisely this direction.

${ }^{8}$ Collins et al. (2013), 581-2; GiBBons (2018), 92ff.; Moseley (2013).

${ }^{9}$ Minter, on the other hand, reserves this term ("visualizer") for hardware or software creations that do not accept user input, and emphasizes that what 
The Jaguar CD was not the first consumer music visualizer. Indeed, the Atari Corporation had already developed an electronic visualizer unit for the home consumer goods market-the Atari Video Music (C240)-as early 1976, thought it was neither especially sophisticated nor popular. ${ }^{10}$ Nor was the Jaguar CD the first home video game console to include an music visualizer. That dubious honor goes to the 3DO, a CD-based entertainment system first manufactured in 1993 that included a visualizer system that allowed its users to choose one of four lurid preset visualizations driven by the sounds of an audio CD that had been inserted. ${ }^{11}$

However, the present focus on the Jaguar CD VLM is motivated by the fact that it marks a milestone on the entry of real-time music visualizers into the home, via the by-then-well-established market for home video games. Furthermore, as Jeremy Wade Morris has noted, there may be an even more intimate link between Minter's software and these commonplace visualizers:

Winamp's visualizations own a large aesthetic debt to work like Minter's and that of other demoscene participants. In Minter's own account of the history of visualizations, he describes a run-in with a Nullsoft employee "who apologized for 'borrowing' the techniques I'd used on the [Atari] Jaguar VLM for their own visualiztaions.” (MORRIS, 2015 : 55)

The VLM's strategies of music visualization, which are discussed in more detail below, anticipate those used the software that mediated the experience of hundreds of millions of listeners as they consumed MP3s in the age of Napster, but also reflect an interest in techniques of video art already well established by

distinguishes his lightsynths from such visualizers is precisely their interactivity. The reality of multimedia technology is messy and resists taxonomies. For scholars of multimedia, it is probably more productive, instead, to speak of degrees of interactivity, audioreactivity, and autonomy in the techniques and interfaces of music/sound visualizers, emphasizing both their family resemblances and dissimilarities, than to draw bright lines between them and balkanize their study on the basis of termology.

${ }^{10}$ The Atari Video Music has received little academic scrutiny, though see the cursory description in Bogost (2011). Cited in GiBBons (2018), 92. Online videos published on YouTube provide adequate technical overviews of the device and its limited capabilities. See, for example, (RetroTech: Atari Video Music - The Migraine Machine, 2017); (Ben Heck’s Atari Video Music Teardown, 2016).

${ }^{11}$ For more on the 3DO, a technically innovative system that was nevertheless a commercial failure, see WOLF (2008), 162-163 
1995. Minter's work thus may constitute a link between these pre-digital, midcentury, non-commercial experiments and the fully commoditized packaging of the same techniques as visualizer plugins for PC media player software in the 2000s.

\section{Senses of "hacking"}

No longer conferring a specific sense of technical ability with cutting-edge computer hardware or software, as it might once have meant to the wizards of MIT profiled by Stewart Brand in his influential Rolling Stone feature (1972), the term has become frustratingly nebulous. The word "hacker" rarely occurs today without some constraining modifier. Perpetrators of data breaches are reported in the news as "malicious" hackers; penetration testers must be "Certified Ethical" hackers before they can be hired by standards-compliant businesses; less-scrupulous enterprises may hire "grey" or "black hat" hackers to do their dirty work; "self-described" or "self-proclaimed" hackers are dismissed as fantasists.

Gisle Hannemyr has identified at least three distinctive (if not entirely distinct) senses in which the word "hacker" is used, and, drawing on Steven Levy's Hackers (1984), identifies some definitive aspects of the "hacker attitude". According to Hannemyr, hackers tend to "reject hierarchies, mistrust authority, promote decentralization, share information, and serve [their] community" (HANNEMYR, 1999). In any event, neither prescriptivist pleas for a wider meaning to the word hacker nor nostalgic attempts to restore some lost, less complicated sense of the word, make for a theory of hacking: what it is that a hacker does.

One ramified account of such a theory is outlined in Mackenzie Wark's A Hacker Manifesto, a thoughtful attempt to raise class consciousness in the participants of a newly-identified struggle between the "hacker" and "vectoralist" classes (WARK, 2004). Corrado Morgana, glossing this central opposition in an introduction to a brief but striking collection of short writings by avant-garde video game artists, explains that hackers possess

a mentality that chooses to challenge conventional knowledge and behavior. Wark refers to factors which steer convention and behavior as "vectoral." These vectors, such as property, information, and capital, are steered by "vectoralists" who would guide behavior for economic gain or other institutional control by fostering predictable behavior. The artist that thinks games questions, juxtaposes, reinvents and challenges these vectors (MORGANA, $2010: 8$ )

The present focus on a piece of consumer entertainment technology and its extended reflection on the multiple senses in which it may be considered to be a 
site of "hacking" - here focusing on Hannemyr's "hacker ethic" (after Levy and others) and Wark's more explicitly political analytic-reminds us that even consumer devices in the contemporary domestic milieu are susceptible to being hacked, with all the liberatory potential that that might entail.

More often used to conjure up extra lives, immortality, or gameworld wealth beyond imagination, David Surman has claimed-concisely and convincinglythat cheating in video games is "hacking for the masses." (SURMAN, $2010: 77$ ) Like hacks in the broader technological community, video game cheat codes also function as kind of cultural capital in gaming communities. Knowing cheat codes imputes insider status to the holder of that knowledge. Surman describes an interactive game level editor hidden in Sonic The Hedgehog (1991) that is revealed by inputting a similarly cryptic cheat code. Identifying what Hannemyr and others theorize as the hacker ethic in action, Surman writes that

[these] players vied for an opportunity to penetrate through the surface of the gameworld, to claim leadership and advantage, and wrestle control of the game from the orthodoxies of standard play (SURMAN, $2010: 75$ ).

The conception of hacking as it is invoked by Hannemyr and Wark, then, is a normative one. "Hacking" is less a virtuosic display of skill or proficiency with a particular technical system (the stereotype has the hacker at the keyboard of a programmable computer), and more of a stance with regard to how one ought to act when confronted with technology. It is, perhaps above all, an ethic of curiosity about how things-socially as much as technologically-might have been otherwise. Like accessing the hidden menu in the VLM, cheating in video games does not require a particularly high level of technical involvement; indeed, the vast majority of video game cheaters simply consume cheat codes or download scripts to tamper with games whose implementation they themselves do not fully understand. But their interest in doing so, as Surman points out, is not a chiefly technical one: videogame cheating is contiguous with the hackerly predilection for demystification. So much is in evidence, as we will see, in "YaK Quick's Guide to VLM Hacking”, the how-to guide documenting how Minter had left a hidden menu inside the VLM for users who shared precisely that impulse.

\section{Evidence base}

In this article, I draw on two principal kinds of primary source to trace the ends to which the FFT is put in Minter's software: online discourse about the VLM and the code for the VLM itself. Fist, the website for Minter's software development firm Llamasoft provides a valuable overview of the visualizer, Minter being most 
intimately familiar with the VLM concept over the many decades of its history. A glimpse into the former community of players that clustered around the Jaguar and the Atari Corporation more generally, can also be gleaned from the online archive of the rec.games.video.atari newsgroup. More ad hoc forms of commentary are no less useful, despite their register: blogs and retrogaming forums are valuable sources of insight into the Jaguar $\mathrm{CD}$ and Atari more generally. ${ }^{12}$ One online community of note is the now-dormant but once surprisingly vital community of developers of "homebrew" games and software for the Jaguar. Their appetite for technical documentation and code has led to service manuals, part lists, schematics, and even source code being made available online. ${ }^{13}$

The ROM dump that includes the VLM code cannot be executed on today's personal computer-the code is written in machine code, a low-level, nonportable type of code that is targeted to run on the specific constellation of microprocessors that jointly make up the Jaguar and the Jaguar CD. In this way, the Jaguar CD exemplifies a "platform": a combination of software and hardware with a particular set of affordances to developers and players (BOGOST and MONTFORT, 2009). Fortunately, an emulator, a program that carefully simulates the hardware of an obsolete computing platform on a modern "host" computer, can be used to experience the VLM-even absent the rare and expensive hardware. I built up my functional description of the VLM by using just such an emulator to re-run the BIOS on my laptop, playing with an almost functionally identical (if remediated) simulation of the VLM. ${ }^{14}$

Importantly, however, I also chose to study the VLM in vitro: in addition to emulating the original gameplay environment, we can look at the code itself, using freely and widely available software tools designed to inspect the content of executable files. Inside otherwise inscrutable machine code can be found

${ }^{12}$ For example, AtariAge (https://atariage.com/index.php), AtariForum (http://www.atari-forum.com/), JagCube (http://jagcube.atari.org/).

${ }^{13}$ At the Atari Document Archive (https://docs.dev-docs.org/).

${ }^{14}$ For a discussion of some of the limitations of emulation and attempts to resolve them, see NEWMAN (2012), 140-149. For the results presented here, I used v.0.06b of the t2k.exe emulator (for Windows) and the Jaguar CD BIOS file (MD5 checksum: 77cd95c7ad06a39f4c59995094aa10f9) available on Minter's website at http://www.llamasoftarchive.org/oldsite/llamadloads2.html. Minter considers this material public domain, writing at:

http://www.llamasoftarchive.org/oldsite/llamasoft/readme.txt. 
plaintext code comments, metadata, and--as we will see-hidden, undocumented software features. Retracing the sequence of events in which I myself came to understand the VLM, we first consider the visualizer's source code in "Following the code", and then, in "Polling rec.games.video.atari", turn to the deeper insights into the software that were gained from the online community of Jaguar aficionados.

\section{Following the code}

Consider a bit-for-bit copy of the contents of the Jaguar CD's BIOS. The BIOS, standing for Basic/Input Ouput System, is the software that is loaded almost immediately upon a console's being started for the first time. Since code like this that performs this function is not intended to be changed by the user and is "burned" semi-permanently into a EEPROM chip on the console motherboard, it is often called "firmware", alluding to its seeming at times like hardware, and at times like software. Such a bit-for-bit copy of game data are sometimes called ROM files-ROMs for short. ROMs are the digital records of obsolete and discontinued games that fuel the retrogaming hobby: without these software copies, retrogamers would have to own or have access to an actual console and a physical copy of the game media for every old game they wanted to play. So it goes for researchers, too.

Opening the ROM file for the Jaguar CD firmware (which, as we know, includes the VLM code) in a regular text editor spews out mostly non-humaninterpretable garbage. However, machine code may contain byte sequences that can be interpreted as potentially human-readable alphanumeric characters. Inside otherwise inscrutable machine code, we can find code comments, metadata, and--as we will see-hidden, undocumented software features. I used the open-source software tool strings, part of the standard toolkit of the reverse engineer, to check the BIOS for any traces of human-readable text. Although much of the output remains inscrutable, at least in the first instance, the first string to pop out of the noise is the following statement:

Virtual Light Machine v0.9//(c) 1994 Virtual Light Company Ltd./Jaguar CD-ROM version/(c) 1994 Atari Corporation//FFT code by ib2/Grafix code by Yak/ c100:

This message proclaims the identity of the code that follows it in the BIOS file: "Virtual Light Machine v0.9". Copyright notices like these are commonplace in software: in the event of a dispute, an author's claim to copyright can be supported by pointing to the existence of these marks. This banner also reveals 
two interesting nuances of attribution: one relating to intellectual property (IP), and one of the division of software labor. First, the Jaguar CD-ROM "version" is claimed by the Atari Corporation, while the IP for the VLM concept itself is claimed by Virtual Light Company, Ltd., a UK-based limited liability corporation founded by Minter. Such a distinction attempts to give juridical support to Minter's firm belief, discussed above, that the VLM is a cross-platform concept: a legal claim that the VLM “idea” is not tied to its instantiation in any one particular game system.

The second distinction tells us which coders were responsible for different parts of the VLM: "FFT code by ib2/Grafix code by Yak". Yak is perhaps Jeff Minter's most frequently preferred pseudonym; ib2, on the other hand, refers to Ian Bennett, one of Minter's former colleagues at the semiconductor firm, Inmos, that fostered the development of the VLM in the late 1980s. Both Minter and Bennett's handles are three characters long, a practical nod to the character limits on arcade cabinet high-score tables. As the code attests, Bennett was responsible for the FFT code in Minter's visualizer, a three-letter acronym that stands for Fast Fourier Transform.

The Fourier transform is a mathematical technique for decomposing a complicated time-varying signal-say music-into an equivalent form, as a mixture of simpler, periodic sine-wave components. Applied to recordings of simple monophonic sounds performed by musical instruments (acoustic or otherwise), it reveals how complex timbres resolve into a fundamental pitch and a distinctive pattern of overtones or partials. It can also be used to analyze more complex recordings, providing a rudimentary parsing of polyphonic recordings into a segmented auditory scene, separating out the musical activity of low-, medium-, and high-pitched sounds into components that can be analyzed and processed separately. In turn, the results of such an analysis may be used to trigger and modulate the visual events in a musical visualizer.

Though purpose-built spectrum analyzers were available from the 1950s onward, a set of mathematical and computational optimizations that were perfected in the 1960s that became collectively known as "fast" Fourier transform (FFT) algorithms allowed for the practical spectral decomposition of live signals by digital computers (DEERY, 2007). The first implementation of an FFT algorithm is usually credited to James W. Cooley and John Tukey for work described in their 1965 publication "An Algorithm for the Machine Calculation of Complex Fourier Series.” (COOLEY and TUKey, 1965) he origins of the so-called Cooley-Tukey FFT 
algorithm is a particularly interesting one $\cdot{ }^{15}$ Remote sensing of the seismographic signatures of nuclear detonations allowed monitoring organizations and national geospatial intelligence agencies to verify the behavior of parties to treaties like the Limited Test Ban Treaty, which was signed in 1963 and placed constraints on the testing of nuclear weapons in a drive toward nuclear disarmament (BISHOP, 2016). To this day, the algorithm described by Cooley and Tukey remains a popular and robust FFT algorithm; Ian Bennett's implementation of the FFT included in the VLM code shares the key features of the Cooley-Tukey algorithm.

Like all modern video games consoles, the Jaguar is composed of many specialized integrated circuits, which can be broadly divided into the main central processing unit (CPU) and two main co-processor systems-the latter two co-processors were nicknamed Tom and Jerry. Their main areas of responsibility were video and sound output, respectively. Bennett chose to write the FFT computation routine for the digital signal processing (DSP) microchip inside Jerry because it provided some special features intended for such computations that the other microprocessors on board the Jaguar did not. ${ }^{16}$ These are specified in the instruction set for the DSP, the set of possible basic computational operations defined by the computer system to which all software written for the Jaguar CD will ultimately be reduced.

Instruction 48, MIRROR, permutes the content of a memory location, which, as the technical documentation for the Jaguar notes, "is helpful for address generation in Fast Fourier Transform (FFT) operations." (BRENNAN, DUNN and MATHIESON, $2001: 10$ ) Furthermore, given that this instruction is implemented in the physical design of the chip itself, the MIRROR instruction exemplifies how material conditions in silico afford certain kinds of optimization, and in turn, the use of the FFT. In this way, material features of the Jaguar system itself afford the FFT and these features are determined prior to the creation of the VLM software. Such properties lie at the level of the platform and bottom out as material features of the microchip processors themselves.

Further into the BIOS code are the following lines, which contain dozens of phrases whose conspicuous absence from the main VLM user interface gives rise to the question: if these strings are not intended to be visible by the user during

${ }^{15}$ See ANSCOMBE (2003), 299-300, reporting a conversation from 1995.

${ }^{16}$ Based on my review of the assembly code targeted for Jerry, which has been made available online. 
normal gameplay, what explains their inclusion in the code shipped to with the Jaguar CD?

$\sim g 1: 20:$ Bank>

$\sim \mathrm{g} 1: 20$ :

@ g1:1:Edit Mode e3:4:

@ g1:1:Editing: Effect $\sim 3: 4$ :

@ g1:1:Editing: Symmetry Generator e3:3:

@ 91:1:Editing: Digital Video Feedback e3:3:

(@ g1:1:Editing: Wave Plotter e3:3:

@ g1:1:Spectrum and Triggers e3:3:

@ g1:1:Trigger Settings e3:3:

@ g1:1:Adjust width using joypad

It turns out that the hidden features of the VLM insinuated by these lines of code are only unlocked when the player inputs an undocumented sequence of controller inputs that are highly improbable in regular gameplay. A series of posts by Minter to the rec.games.video.atari newsgroup in 1996 explain precisely how to cause these menus to appear, describing officially undocumented controller actions that activate a hidden feature, a set of actions that even casual gamers would recognize as cheat codes. Software features such as this, that are hidden behind obscure game behaviors, are sometimes called easter eggs, "digital objects, messages, or interactions built into computer programs by their designers [...] intended as a surprise to be found by the user, but [...] not required in order to use the program." (NOONEY, $2014: 165$ )

In his methodological guide to the study of game music, Tim Summers describes a mode of collecting analytical data he calls "reactionary play": playing against the grain, taking in-game actions or adopting strategies unsanctioned-or perhaps even unimagined-by the game designer. Doing so can probe the limit cases of the game with the hope of revealing its musical mechanics, telling us under what circumstances are musical cues triggered, cycled, or muted (SUMMERS, $2016: 12$ ). It should be noted that even extended reactionary play, following Summers, would be highly unlikely to take us "behind the curtain" of the VLM: the cheat codes required to get to the hidden menus remain "out of reach" within the gameworld. Of course, Summers takes pains to enumerate alternative "sources of data" for the study of video game music including, importantly, the game engine code itself. Thus to Summers's impressive and useful inventory of means to understand the music-video game relations we add cheats and hacks: (semi-)clandestine ways of interacting with video games that 
violate the norms of regular, expected gameplay, but fall short of a completely forensic-and technically non-trivial-deconstruction of the software.

\section{Polling rec.games.video.atari}

Without the benefit of the synoptic view on the digital artifact provided by these tools however, owners of the Jaguar CD in 1995 turned to another source in search of knowledge about their new purchase: the rec.games.video.atari Usenet group, a distributed message system that relayed plain text messages posted to the group between subscribers. On November 14, 1995, less than two months after the release of the Jaguar CD, a user named Julienne Rha posted to rec.games.video.atari:

is there any way to customize the banks?? [...] could ANYONE please tell me if any eastereggs exist and if so, how to activate them (RHA, 1995).

This is the first indication on the newsgroup of suspicions that there might be hidden content in the visualizer part of the Jaguar CD firmware. Rha's desire to customize the preset visualizations is echoed by other subscribers to the news group. We have already seen how the VLM is distributed along with the Jaguar CD operating system, as firmware, in a read-only EPROM chip, and is therefore prohibitively difficult for most end-users to update. ${ }^{17}$ The distribution of the VLM in the device firmware, a tactic that Minter hoped would secure as wide an audience as possible for his visualizer, necessitated just such a trade-off: the VLM would be shipped to every owner of the Jaguar CD, but once the unit left the factory, the software could not be modified. This raised the stakes for a question like Julienne Rha's. Since no new features-for example, the desired ability to customize the preset banks-could be added to the VLM by its developer, it was important for players to know exactly what was there, and, if it was hidden, how to uncover it.

Then, on March 18, 1996, Minter posted to a thread looking for further clues (a thread that had, in the meantime, devolved into a flamewar about a completely different game):

Okay, to get this thread back on topic and to give you a starting point: Try 1,3,star,zero. That's just the start though (YAK (JEFF MiNTER), 1996).

${ }^{17}$ At least without replacing the chip with new hardware or by using other specialist tools. 
Entering Minter's pattern reveals the first of two hidden menus: the "Spectrum and Triggers" menu (Figure 4). This screen visualizes the beating heart of the VLM: the real-time decomposition of the music into the frequency-intensity domain that the FFT algorithm supplies. Like a graphic EQ visualization, the height of each bar represents the magnitude of frequencies within some fixed range, or bandwidth. The midpoint of each range increases in frequency from left to right. In other words, low-pitched sounds make peaks on the left; highpitched sounds are peaky on the right.

[FIGURE 4 04_spec-trig.png]

Triggers are defined by a combination of parameters that dictate what shapes of the spectrum cause the visualizer to change in response to the music. ${ }^{18}$ For each trigger, the "Spectrum and Triggers" menu exposes just two parameters, which pertain to the audio step: total customization of the visual effects was, at this stage, out of reach to the participants in the newsgroup. According to Scott Lawrence's analysis of the trigger conditions, each trigger fires or otherwise modifies just one visual effect, according to a set of rules that results in "a situation where loud sudden things will cause a trigger, however constant noises will probably not cause triggers. Also, quiet noises will never cause triggers.” (LAWRENCE, 2009)

The ability to control these parameters in the Spectrum and Triggers menu gave players a more fine-grained and more reliable way to tune the VLM than simply adjusting the gain level, a relatively crude and one-dimensional means of controlling the behavior of the visualizer. Tweaking the parameters for each trigger using these menus adjusts these policies so that the graphics appear to synchronize to musical activity in particular parts of the spectrum, based on generic or expert expectations about how particular songs will sound. Activity in different parts of the spectrum may therefore be loosely correlated to musical content: the low frequencies of the bass drum or a synthesized bassline might be set to trigger one visual event, where the higher frequencies of an instrumental line or wide-band sound effect will activate another.

Minter understood certain presets to be better suited to certain tracks or genres. In the same post that introduced the VLM editor mode, Minter included a listening suggestion:

${ }^{18}$ Each trigger has an associated width (represented graphically by the red bars in the frequency-domain display) and a trigger minimum (represented by a horizontal grey line). 
BTW - for general most excellent VLMing... get that NIN cd with the 5 mixes of 'Closer' on it, select track 3 and effect 4:9... intense or what? (YAK (JEFF MINTER), 1996)

Minter provided updates to a page on his personal website that listed his own listening recommendations, often suggesting VLM preset that he felt best matched each musical selection (MINTER, 1995). Newsgroup users also shared recommendations for suitable matches (MILLAR, 1995). The circulation of such "good fits" shows how a community can implicitly define a set of desirable audio-visual correspondences. These valued correspondences can come not only from the creator (along with the sanction afforded by Minter's authority) but from the player-users themselves. For example, writing in reply to a post titled "What do you like to play on VLM?", Brian J. Geiger answered:

music like Erasure (especially their singles) and Music for Boys by the Pet Shop Boys works really well with that module [i.e. VLM effect preset], because it starts out just showing the sound wave, then when the real music starts, the screen figuratively explodes (GEIGER, 1995).

Users did not require a game disc or cartridge to load the VLM; indeed, the visualizer is only accessible when a game disc is not inserted. As such, it is predisposed to work with a particular kind of format-the audio $C D$, though some explored the possibility of linking the output of other playback systemstape decks, LP players, or radios-to the VLM, to widen the range of audio sources with which it could work. For example, a newsgroup poster requested schematics for the device, who was "tring to connect my jaguars vlm system into my sterio so i can play tapes,records etc through the vlm chip." [sic] (BOLSER, 1996)

Such "interpersonal interactions mediated by a game", as Karen Collins has intimated, also exemplify a kind of interactivity, one that "take[s] place on a much vaster and temporally longer scale" than the instant-to-instant interactions that determine how video games behave in the moment of play (ColLINS et al., 2013 : 575). A more generous understanding of interactivity both exceeds and complements the control-feedback model that Collins uses to analyze audiovisual relationships in video games. Such digital traces of community of players provide further evidence for the VLM's interactivity, understood both as a characterization of the relationship between the player and the game, but also of those relationships between players, regardless of their spatial or temporal remove. 


\section{Total customization?}

The precise correspondence between trigger and visual effect is more fully determined in later set of menus, along with the actual definition of the graphical content of each effect. As Minter would later write, the Spectrum and Triggers menu "was just a red herring to confuse people looking for the hack"; the true hidden menu that would allow players to customize the preset visualizations was one more step away. Just two days later, Minter posted to the newsgroup with details of how to reveal another screen, the realcomprehensive-visual effects editor. Carefully following Minter's instructions reveals a second set of menus. These menus, shown in Figure 5, allow for even finer control over the visual elements corresponding to each trigger and to design periodic functions of substantial complexity that control how basic elements of the visualization move across the display. Here, players can fully parameterize many of the generative graphical processes that, when superimposed, generate the diverse banks of preset visualizations.

[FIGURE 5 05_subslots-4-9.png]

The Spectrum and Triggers screen clarified the dependency between the analyzed audio spectrum and the visual cues, defining six "triggers" that can be tuned to different regions of the frequency-amplitude domain. Using the next level of menus, we now focus more closely on the fourth setting in the ninth bank of presets (4-9) by visiting the sub-effect slot menu. From this menu, we learn that each individual preset consists in the superimposition of up to six distinct visual effects which are optionally passed through a "symmetry generator", which is parameterized in another sub-menu, shown in Figure 6. Despite its emergent visual complexity 4-9 was a relatively straightforward preset composed of just two such sub-effects: Draw Spectrum As Intensities and Digital Video Feedback area. Of the two, it is the digital video feedback effect that creates the ever-shifting play of multiple forms that characterizes many of the VLM's presets. The technique was simple but effective.

[FIGURE 6 06_further-menus.png]

Thus, over the course of these two messages, Minter had revealed to the newsgroup a pair of "cheat" codes: a set of controller inputs that cause the game to behave in undocumented ways. Some days later, on ch 31, Minter summarized his revelations in a complete guide to the VLM in a newsgroup post with the subject line: "YaK's Quick Intro to VLM Hacking", the title invoking the ethic of 
subversion and détournement familiar to the patient readers of certain mid-90's bulletin boards and newsgroups. Minter's guide comprehensively detailed how to access the hidden menus, and what could be achieved in them.

Minter's three answers to the rhetorical question-why?-explicitly address the existence of the hidden menu in terms that recollect Hannemyr's sketch of the hacker ethic:

Because it’s fun. (YAK (JEFF MINTER), 1996)

First, and crucially, Minter offers the ludic motivation. Minter's commitment to fun supersedes any professional commitment to ship fully-tested and stable software. Minter much prefers something broken but fun to something perfect but boring.

Because if you're interested, you can find out a lot about how the VLM works.

Next, hacking the VLM feeds an intellectual curiosity. As Minter explains,

[Draw Spectrum As Intensities] basically plots the output of the FFT through the symmetry generator. On its own, or combined with DVF [digital video feedback], this is a good setting to use for effects that you want to be precisely audio-reactive.

The technique of digital video feedback (DVF) is the visual analog of an audio echo effect: a seed visual form is generated and immediately duplicated, usually with a rotational and/or translational spatial offset. This image is then recursively fed back into the duplicator, creating a plenum of superimposed copies of the original seed form, interactively collapsing into a singularity. The kaleidoscopic effect is familiar to anyone who has stood in between two facing mirrors, or pointed a home-video recorder or a webcam back at the monitor displaying its output.

Feedback techniques were close to axiomatic for 1960s video art for the early New York-based explorers of the potentials of video art. Woody Vasulka recalls that "in 1969, the first common artifact of [the video] medium was a video feedback. It became overnight everyone's art [...] [.] It was so easy to make.” (WILSON and MELEGA, 1981 : 9) DVF techniques, and the closely-related mirror symmetry effects, like those worked out and exposed in the VLM's hidden menus are a recurring design motif in Minter's video game work. ${ }^{19}$

${ }^{19}$ See, for example, his early Psychedelia (1984) through to the ever shifting background art of Tempest 3000 (2000), Space Giraffe (2007), and, more recently, Polybius (2016) as well as his work on the Xbox 360's built-in music visualizer, 
Lastly, Minter characterizes the hidden menus as a means to empower the user with the tools to create their own effects, so that they might exceed Minter's self-confessed creative limitations arising from a badly-timed illness:

Because a lot of the default FX are fairly nonoptimal, largely 'coz at the time I was designing the banks I had pneumonia and was feeling like shit, and I always find that I make better FX when I feel good.

Minter's perfectionism aligns with the hacker ethic, which has the hacker "either attempting to right those parts of [their] program that are not functioning as smoothly as they might, or, alternatively, adding elements that are designed to improve what is already there." (DARLEY, $2000: 23)^{20}$ In his guide, he reinforces the provisional nature of the code:

the edit interface is buggy and pretty user-hostile; it's got no error-checking and it's possible to crash the VLM [...] Hell, it was never intended for end-users.

Sue Morris has observed that those first-person shooter games that feature level editors, which allow gamers to design and sometimes share custom gameworlds or "maps", should be seen as "co-creative media". Just as in the case of the VLM, [n]either developers nor player-creators can be solely responsible for the production of the final assemblage regarded as "the game", it requires the input of both (MORRIS, 2003).

Players of the VLM are invited to engage with and modify, however temporarily, a piece of software whose limitations are freely acknowledged by its creator. By including the hidden preset effect editor menus in the Jaguar CD VLM, Minter deliberately facilitated playful co-creation on the part of his players, leaving the back door open so that curious users could transcend the game's functional and aesthetic limitations by using a tool originally intended for the sole use of software developers. The circulation of the cheat codes that summon up these secret menus, in Surman's words, afforded a "hacking for the masses" that opened Minter's VLM to extension and elaboration beyond the ossified state in which commercial contingencies determined that it should leave the software development workshop.

Above all, Minter's hidden menu is a solicitation to interact with this audiovisual toy and to implicate savvy users in a process of software co-creation that empowered them to address the software's inevitable lacks. "YaKs Quick Intro",

codenamed "Neon", discussed in more detail below.

${ }^{20}$ In this connection, on the characteristic tool-making impulse of the hacker, see LEVY (1984), $142 \mathrm{ff}$. 
then, is more than just a user's guide to the hack. It is also a statement of purpose, a manifesto: it specifies terms of engagement with the VLM, which privilege fun, curiosity, and above all, the invitation to get involved in the cocreation of the VLM software, through an interface that came with no guarantees about its stability.

But the VLM, and software more generally, is not merely the product of a coproduction of two kinds of putatively human authors ("developers" and "playercreators"), it is an assemblage of actors and actants that is also, in part, nonhuman: the Jaguar CD device, its controllers, the particular audio CD-ROMs that happen to be available to its players, the FFT-specific instructions afforded by its processor architecture, Minter's guide to "hacking” the machine, the Usenet infrastructure through which knowledge of the cheat codes propagated...-the litany is infinitely extensible. ${ }^{21}$ These facts serve as a reminder of the constitutive force of things on multimedia software, troubling implicit and explicit assumptions that human actors dominate the author function when analyzing co-creative media.

This reconfiguration redirects our attention back to the VLM's dependence on the Fast Fourier Transform algorithm. Far-removed from the clinical Cold War logic that motivated the need for a faster Fourier transform, Minter's video game art instead embraces an offbeat, outsider aesthetic. Abandoning realism, he prefers to populate his games with furry animals instead of humanoid playable characters and cartoonish chaos over and above simulated violence. Minter advertised his earliest lightsynth "as a new, non-competitive form of entertainment... no enemies, no killing, just light and colour [..] it was clear that some people just didn't 'get it' at all [...] I guess it was hard to review since it wasn't a game, and all the reviewers were used to was games.” (MiNTER, 2005a)

Reviews of Minter's games, like this review of Space Giraffe (2007), often invoke their psychedelic aspect-the point of reference is often acid or some other psychoactive substance: "the throbbing LSD nightmare that is the background [...] makes this game uniquely aggravating” (AMRICH, 2007; See also, RUGGILL, 2015 : 85-87; WEINEL, 2018 : 130-131.). Minter himself is not entirely averse to coy allusions to the origins for the visual language of the VLM. As he notes on his website:

${ }^{21}$ This position transparently owes its debts to the work of Bruno Latour, though I do not profess to be dogmatic with regard to Actor-Network Theory. In English, see the helpful introduction to many of Latour's ideas in LATOUR (2005). 
I found myself with this stunning little bit of kit which could produce effects I'd never dreamed of (well, I probably had dreamed of them, but not in a legal state of mind) (MINTER, 2005b).

After all, Minter named his earliest published experiment in interactive color games Psychedelia, while the first incarnations of the VLM-the prototypes first developed at Inmos-made their appearance at raves. At one point, Minter's work had featured in live DJ sets by the British house duo The Orb (MINTER, 2005b). Whatever Minter's position on the matter, however, the visual language of the VLM and its contextual association with genres and performance contexts that can be generously described as psychedelia-adjacent, suggests it may productively be read as a counter-cultural supplement to the listening experience.

Yet, as we have noted above, it drew on advances in numerical computing that had been developed in direct response to Cold War demands for global geospatial intelligence collection. This irony could be construed as a quirk-a unintended-but-pleasant consequence-of a fully liberalized market for scientific research that flows, inevitably, into consumer products. Following the more cynical view of Friedrich Kittler, we might view it as further evidence of the systemic influence of military-industrial research on on the entertainment industry. ${ }^{22}$

It is provocative, in conclusion, to ponder FFT algorithm in commercial music visualizers as its own "grand hack", a creative rerouting or redirection (loosely following Wark) of the intellectual innovations emanating from technocracy of wartime scientific research. Away from the battlefield and into the stoner's den, the algorithm becomes neutered and mocked as technology of warfare by being press-ganged into the service of psychedelic lightshows. The ethic of the spirited curiosity that typifies Hannemyr's hacker-exemplified equally well by the erstwhile subscribers to rec.games.video.atari or the contemporary researcher, armed with tools to dismantle obsolete software-is legible both in the momentto-moment play of lights of Jeff Minter's Virtual Light Machine and, especially, in the tactical disclosure of the intricacies of the complex (socio-)technical system that animates them.

${ }^{22}$ Ever-provocative and eminently quotable, Kittler (in)famously remarked that "the entertainment industry is, in any conceivable sense of the word, an abuse of army equipment," KITTLER (1999), 96. Kittler had music in mind: Stockhausen's Kontakte (1958-60) made use of army surplus signal generators. 


\section{Feedback, feedforward}

A later version of Minter's VLM that he developed for the Nintendo Gamecube caught the attention of a product manager at Microsoft, an encounter that ultimately led to Minter's implication in the design of the music visualizer which shipped with Microsoft's Xbox 360 in 2005. Reflecting on the inclusion of his software on a system that witnessed sales in the hundreds of millions of units, Minter writes:

I am happier with it than I have ever been with anything I've created in my entire career. And we got it into the firmware again... Microsoft firmware. [...] At last... after more than 20 years' work, one of my lightsynths is going to reach a decent sized audience. Millions and millions..... \}:-D (MINTER, 2005a)

The market penetration of the Xbox 360 dwarfed the preceding commercial instantiations of the VLM that preceded it: in addition to the Jaguar CD version discussed in detail above, an updated version of Minter's visualizer was distributed in the firmware of a number of feature-rich DVD players manufactured under the Nuon brand. ${ }^{23}$ Unlike the Jaguar CD VLM, no hidden menu or interactive preset editor is included with the Xbox 360. Minter, perhaps, was at last satisfied with a realization of his lightsynth concept on a platform with what he described as "monster shader performance" and "a hell of a lot of grunt", not to mention its impressive audience; during the era of the Jaguar CD, Minter asserted, "consumer-level hardware was simply not powerful enough to really implement such a thing properly" (STUART, 2005).

Nevertheless, in a December 2005 post to the forums at YakYak.org-"Llamasoft baanter and moosings - It's like Deliverance with Sheep"-Minter provided documentation that described in detail how each of the Xbox 360's four controllers can be used to modify and interact with the visualizer; these details were not distributed with Microsoft's console either in the official manual or within the software itself (YAK (JEFF MinTER), 2005). The spirit of “YaK's Quick Intro to VLM Hacking", of implicating the user in the creation of better audiovisual software worlds through customizable software and profligate information-sharing, lived on.

\footnotetext{
${ }^{23}$ Nuon was a short-lived but forward-looking experiment in home entertainment hardware designed in the early 2000s, and Minter was invited to include an updated version of the VLM in a number of units. For more on Nuon, see Moss (2015).
} 


\section{Images}

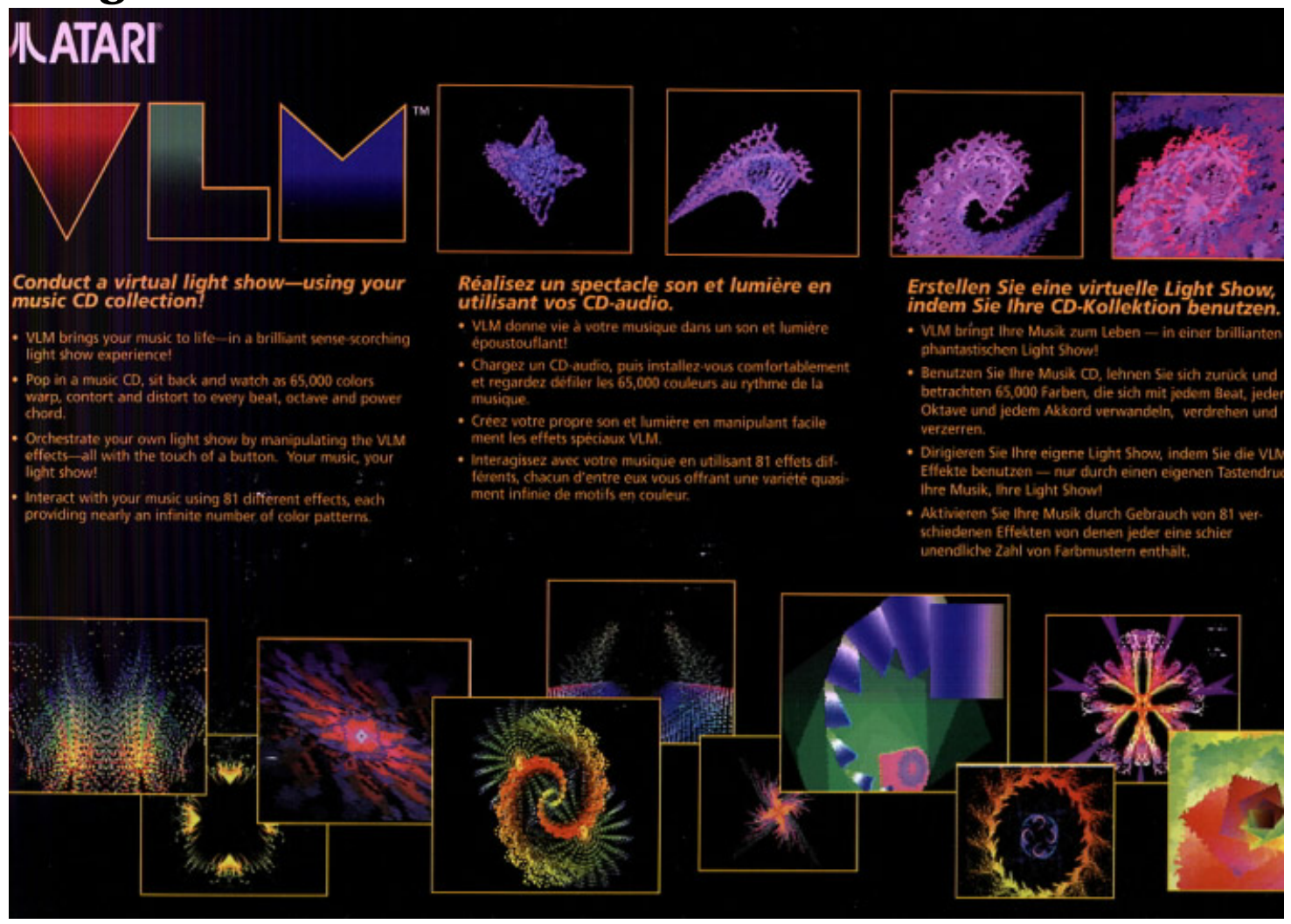

Figure 1: Promotional copy in three languages on the back panel of the original packaging of the Atari Jaguar CD. Source: http://jagcube.atari.org/jaguarcd.html. Accessed 28 October 2018. Copyright: Atari Corporation, Inc. 


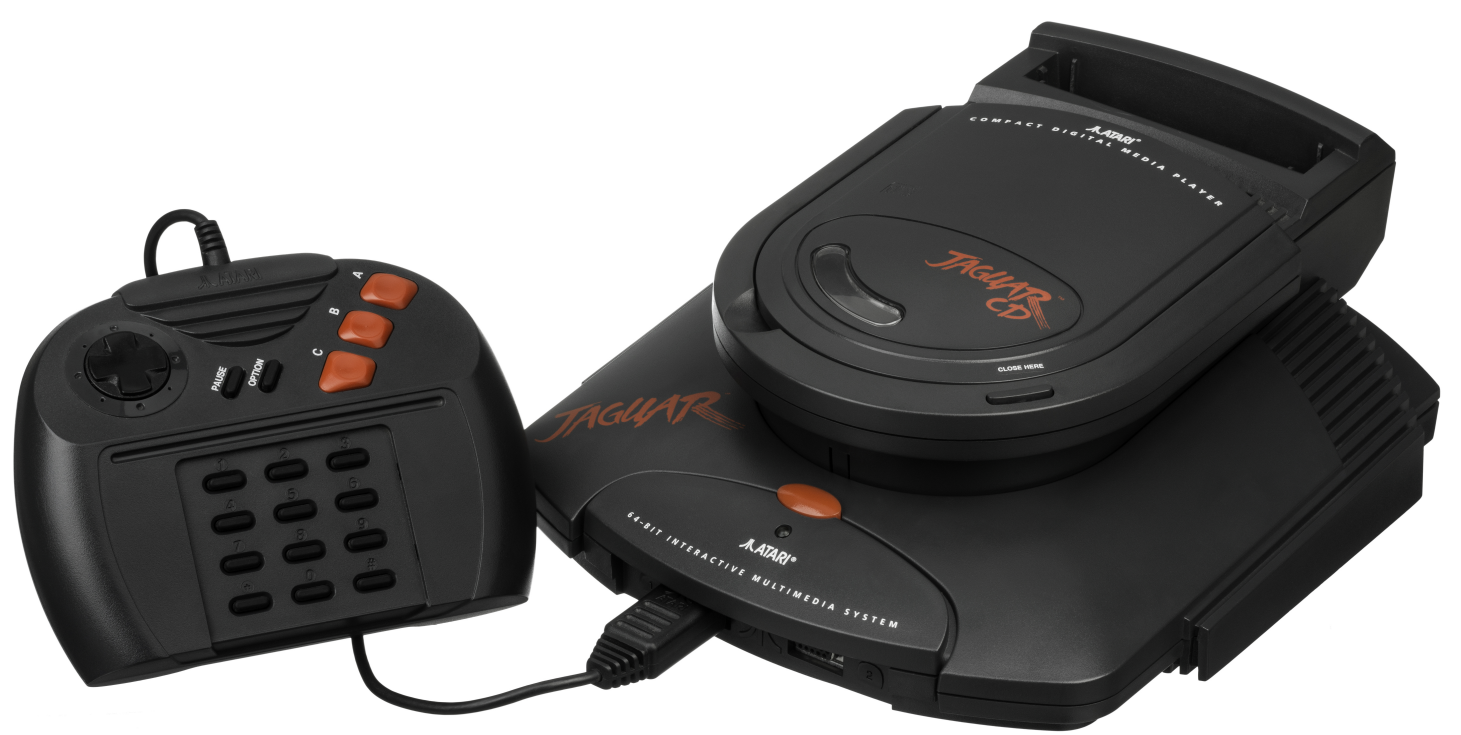

Figure 2: The Atari Jaguar with the Jaguar CD add-on in situ, along with the its idiosyncratic controller. The Atari Jaguar controller featured a telephone-style numeric keypad, which is used in the VLM to switch between visualizer presets. Source: https://commons.wikimedia.org/wiki/File:Atari-Jaguar-CD-wController.jpg. Accessed 14 July 2019. Credit: Released into the public domain by Evan-Amos. 

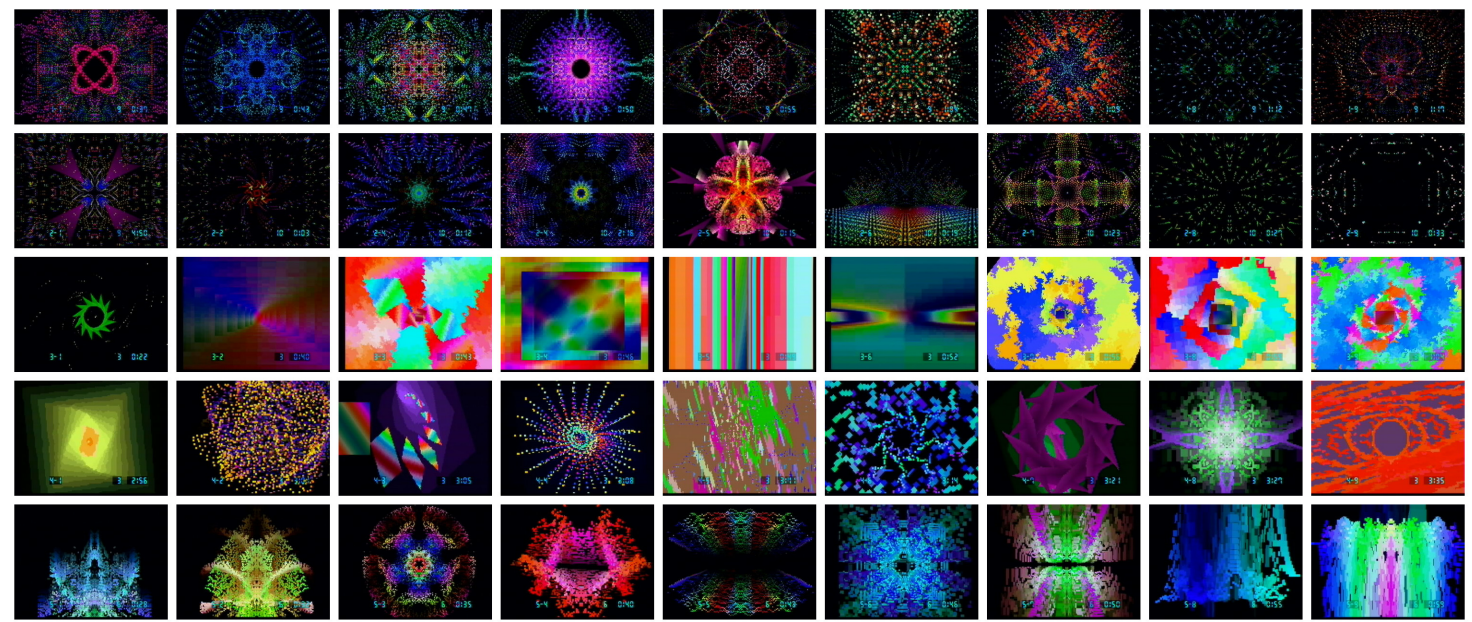

\%
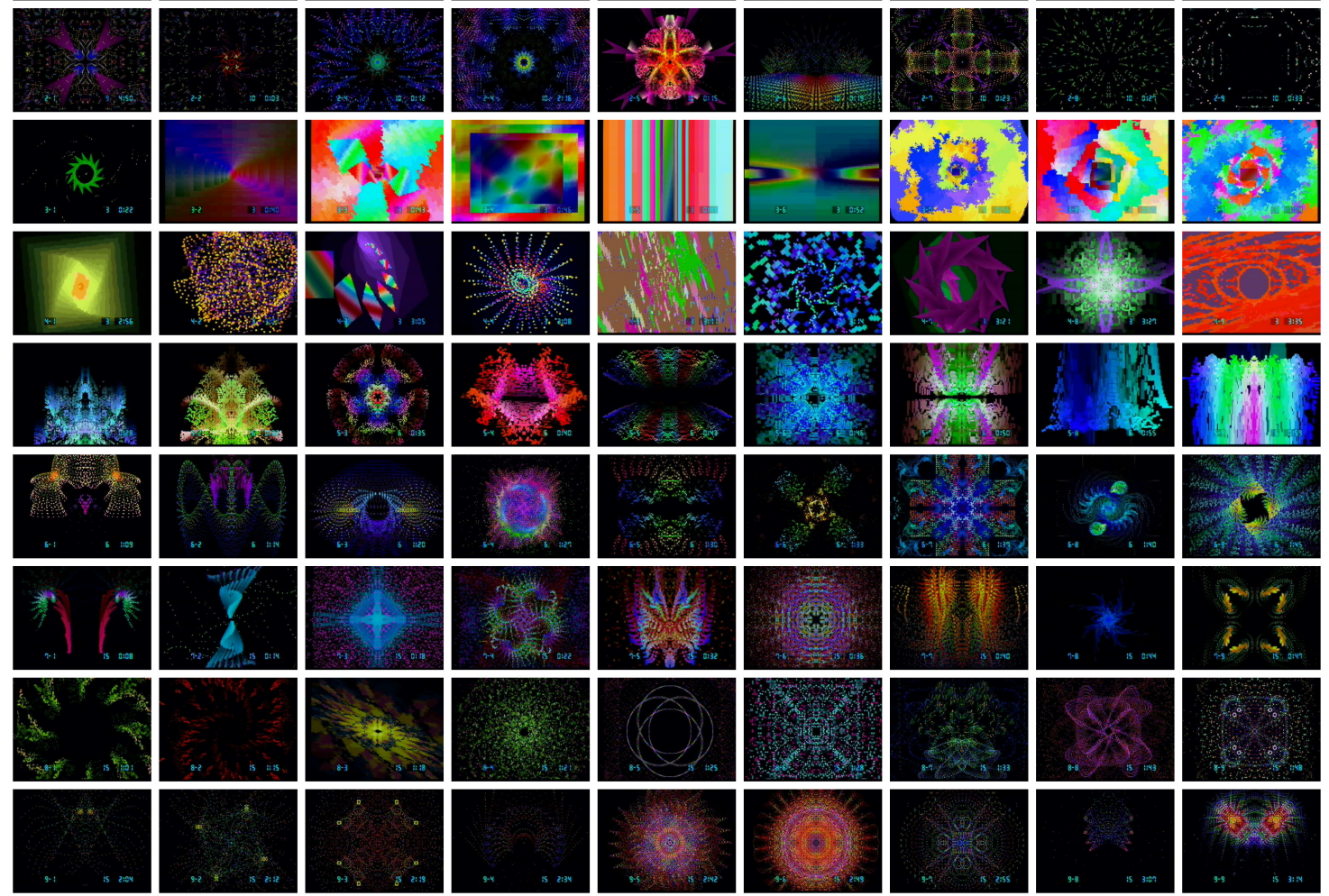

Figure 3: The author's montage of screenshots of each one of the 81 of the preset visualizations provided by the Virtual Light Machine out of the box. Source: http://jagcube.atari.org/jaguarcd.html. Accessed 28 October 2018. Last updated 22 January 2002. 


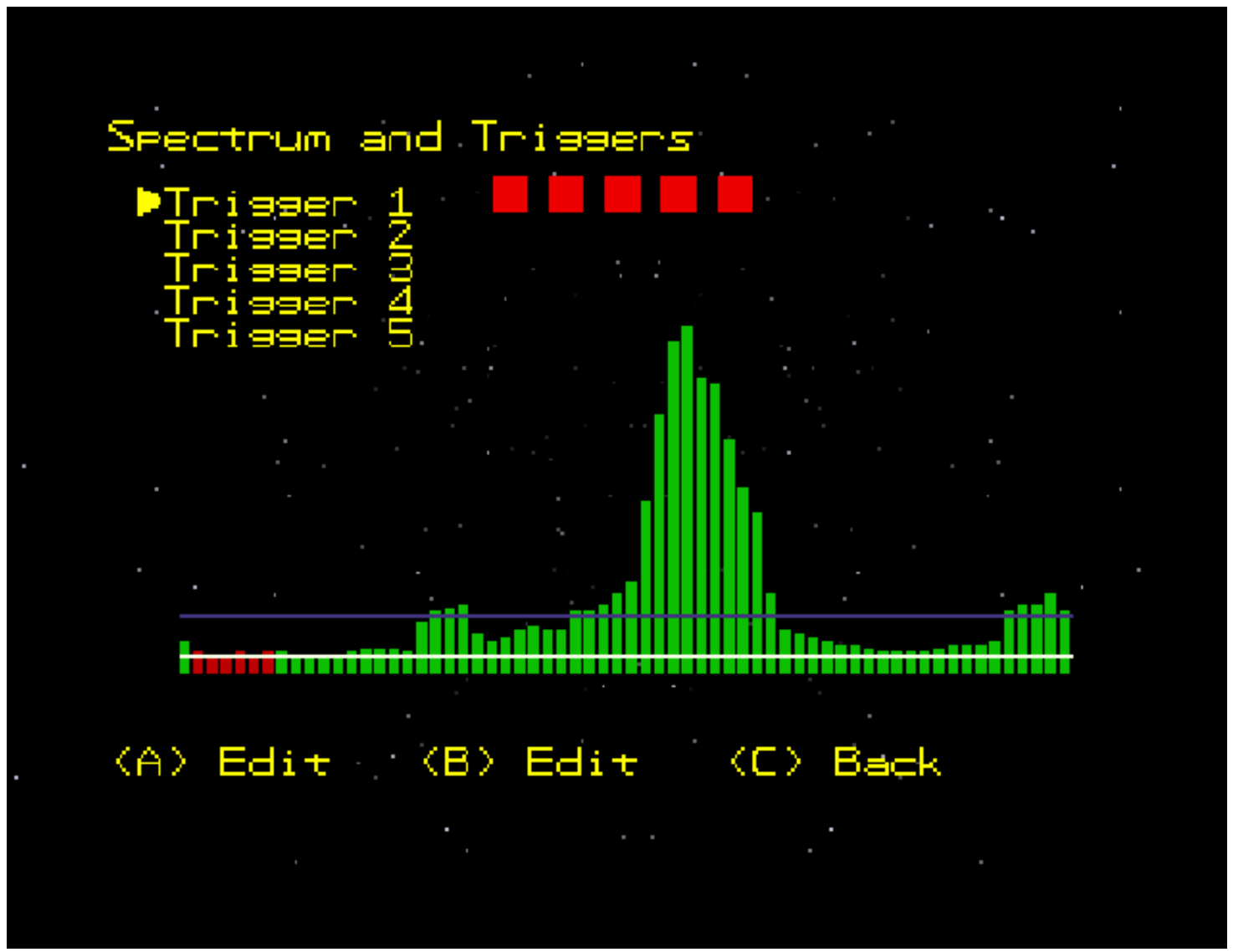

Figure 4: The Spectrum and Triggers menu showing the bars that correspond to the frequency-intensity domain data computed by the Fast Fourier Transform (FFT). Source: Author's screenshot of the t2k.exe emulator running under Linux/WINE. 
Choose a subeffect slot to Edit

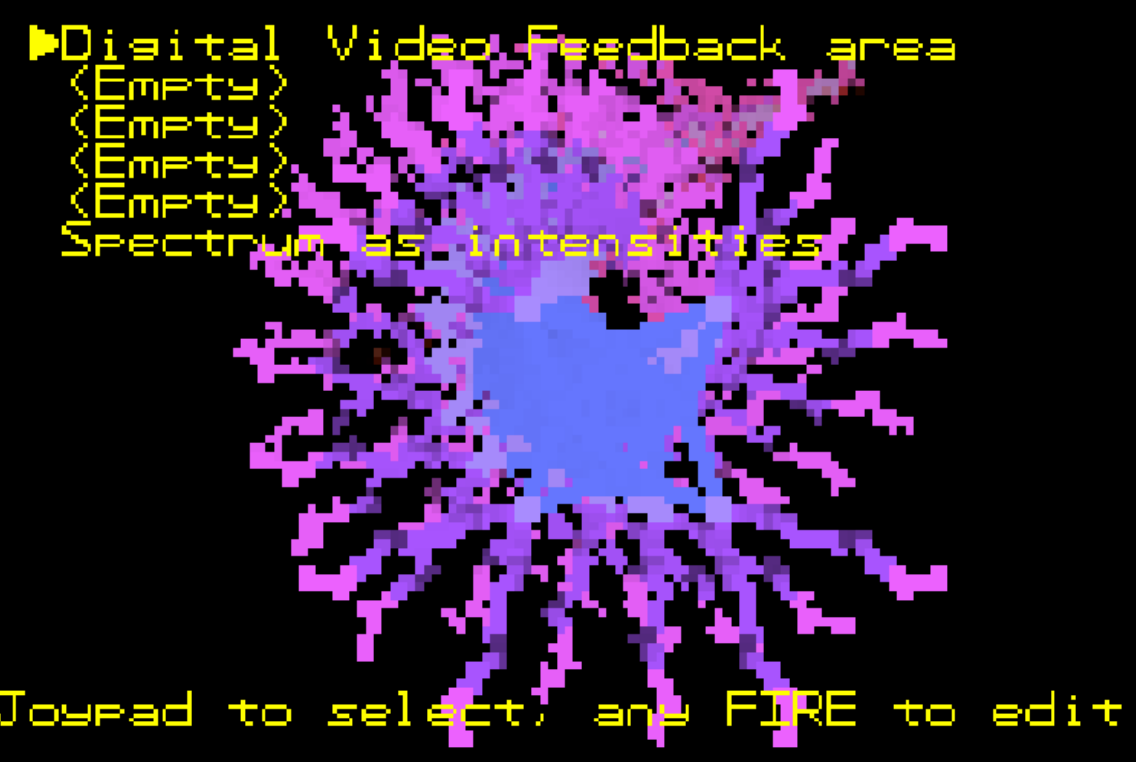

Figure 5: VLM effects editor sub-menu showing the two visual effects which together make up preset 4-9. Source: Author's screenshot of the t2k.exe emulator running under Linux/WINE. 


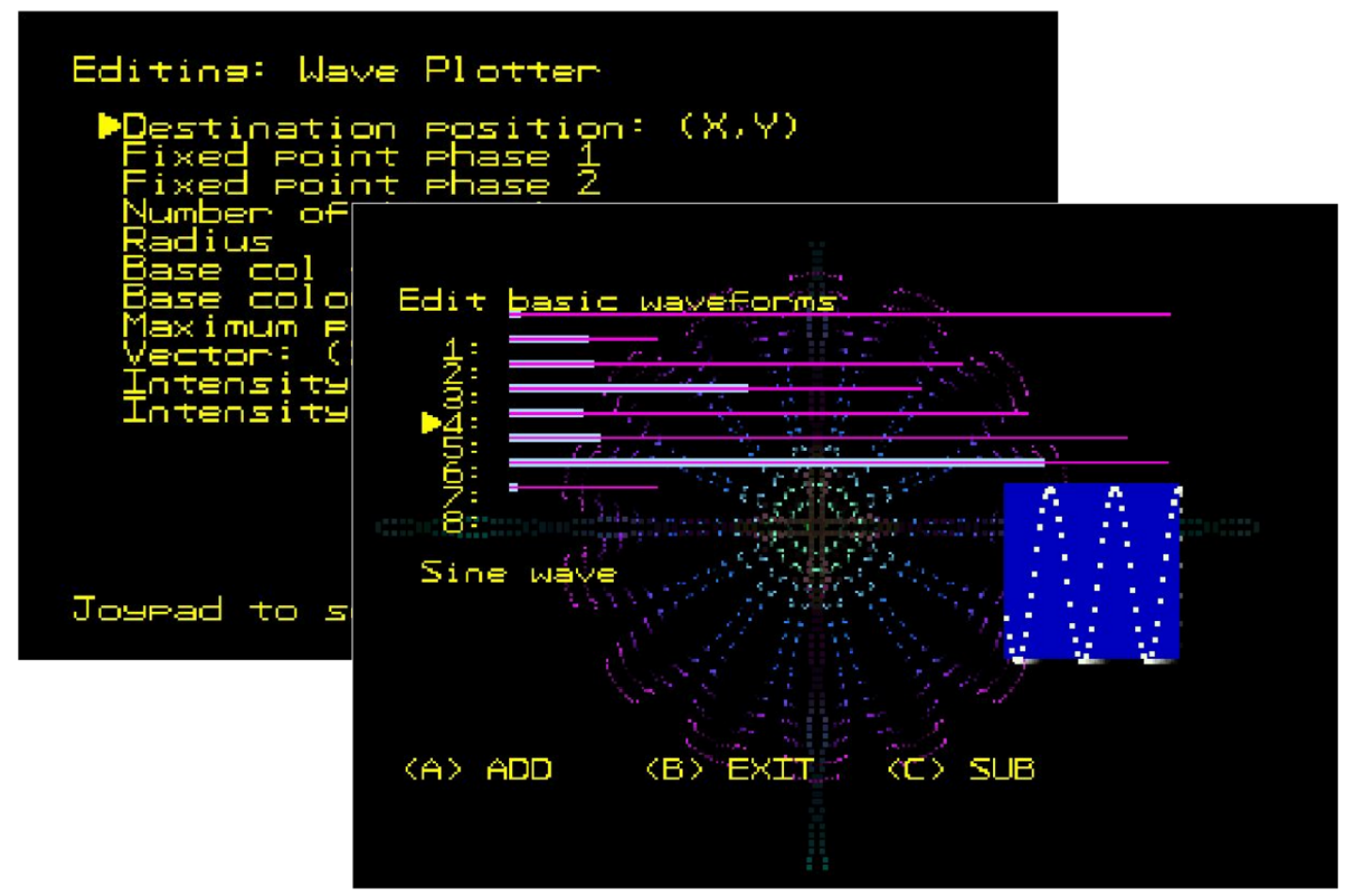

Figure 6: Some examples of further sub-menus inside the hidden VLM effects editor. Source: Author's screenshot of the t2k.exe emulator running under Linux/WINE.

\section{Works cited}

AleXANDER, A. and Collins, N. (2017) 'Live Audiovisuals', in Collins, N. and D'EsCRIVAN, J. (eds) The Cambridge Companion to Electronic Music. 2nd edn. Cambridge: Cambridge University Press, 123-137. doi: 10.1017/9781316459874.009.

Amrich, D. (2007) 'Space Giraffe (review)', Official Xbox Magazine, September. Available at:

https://web.archive.org/web/20091123125836/http://www.oxmonline.com/ article/reviews/xbox-live-arcade/s-z/space-giraffe (Accessed: 29 October 2018).

ANScombe, F. R. (2003) 'Quiet Contributor: The Civic Career and Times of John W. Tukey’, Statistical Science, 18(3), pp. 287-310. doi: 10.1214/ss/1076102417. 
ATARI CORPORATION (1996) Annual report pursuant to Section 13 or 15(d) of the Securities Exchange Act of 1934 for the fiscal year ended December 31, 1995. Form 10-K. United States Securities and Exchange Commission. Available at: https://www.sec.gov/Archives/edgar/data/802019/0000891618-96-000213.txt.

BAIN, M. N. (2008) Real time music visualization: A study in the visual extension of music. $\mathrm{PhD}$ diss. The Ohio State University.

Ben Heck's Atari Video Music Teardown (2016). Available at: https://www.youtube.com/watch?v=INnpnJvDXDg (Accessed: 17 July 2019).

BISHOP, R. (2016) 'Smart dust and remote sensing: The political subject in autonomous systems', in BECK, J. and BISHOP, R. (eds) Cold war legacies: Systems, theory, aesthetics. Edinburgh: Edinburgh University Press (Technicities).

Bogost, I. (2011) How to do things with videogames. Minneapolis, MI: University of Minnesota Press (Electronic mediations, 38).

Bogost, I. and MonTForT, N. (2009) 'Platform Studies: Frequently Questioned Answers', in Proceedings of the Digital Arts and Culture Conference. Digital Arts and Culture, Irvine, CA.

BOLSER, D. (1996) VLM, CAN U HELP!, alt.atari-jaguar.discussion. Available at: https://groups.google.com/forum/\#!original/alt.atari-jaguar.discussion/ gSN6hnQkznY/oNI-lMveQUkJ (Accessed: 9 November 2018).

BRennan, M., Dunn, T. and MAthieson, J. (2001) Jaguar Technical Reference Manual: Tom \& Jerry. Revision 8. ATARI Corp.

ColLINS, K. et al. (2013) 'Implications of Interactivity: What Does it Mean for Sound to be "Interactive”?', in The Oxford Handbook of New Audiovisual Aesthetics. Oxford: Oxford University Press. Available at: http://dx.doi.org/10.1093/oxfordhb/9780199733866.013.0011.

Collopy, P. S. (2014) 'Video Synthesizers: From Analog Computing to Digital Art', IEEE Annals of the History of Computing, 36(4), pp. 74-86. doi: 10.1109/MAHC.2014.62.

COOley, J. W. and TUKeY, J. W. (1965) 'An Algorithm for the Machine Calculation of Complex Fourier Series', Mathematics of Computation, 19(90), p. 297. doi: $10.2307 / 2003354$. 
DARLEY, A. (2000) Visual digital culture: Surface play and spectacle in new media genres. London: Routledge. Available at:

https://ebookcentral.proquest.com/lib/columbia/detail.action?docID=165889 (Accessed: 26 October 2018).

DEERY, J. (2007) 'The Real History of Real-Time Spectrum Analyzers', Sound \& Vibration, January, pp. 54-59. Available at:

http://www.sandv.com/downloads/0701deer.pdf.

GeIGER, B. J. (1995) Google Groups, rec.games.video.atari. Available at: https://groups.google.com/forum/\#!original/rec.games.video.atari/29DEW34zSA s/MxKff90PCH8J (Accessed: 15 July 2019).

GibBONS, W. (2018) Unlimited replays: Video games and classical music. New York, NY: Oxford University Press (Oxford music/media series).

HANNEMYR, G. (1999) 'Technology and pleasure: Considering hacking constructive’, First Monday, 4(2). doi: 10.5210/fm.v4i2.647.

Izvolov, N. (2014) 'From the History of Graphic Sound in the Soviet Union; or, Media without a Medium', in KAGANOvSKY, L. and SALAZKINA, M. (eds), LEVCHIN, S. (tran.) Sound, Speech, Music in Soviet and Post-Soviet Cinema. Bloomington, IA: Indiana University Press, pp. 21-37.

KiTTLER, F. (1999) Gramophone, film, typewriter. Stanford, CA: Stanford University Press (Writing science).

LATOUR, B. (2005) Reassembling the social: An introduction to actor-networktheory. Oxford: Oxford University Press (Clarendon lectures in management studies).

LAWRENCE, S. (2009) Reverse Engineering VLM-1 Triggers, Yorgle Notebook. Available at: http://yorgle.org/vlm/ (Accessed: 18 October 2018).

LEVIN, T. Y. (2003) "“Tones from out of Nowhere”: Rudolph Pfenninger and the Archaeology of Synthetic Sound', Grey Room, 12, pp. 32-79. doi:

10.1162/152638103322446460.

LEVY, S. (1984) Hackers: Heroes of the computer revolution. 1st ed. Garden City, NY: Anchor Press/Doubleday. 
MILLAR, J. T. (1995) Re: What do you like to play on VLM?, rec.games.video.atari. Available at: https://groups.google.com/forum/\#!original/rec.games.video.atari/ 29DEW34zSAs/YCwREWHXzvsJ.

MinTER, J. (1995) In the Toilet. Available at:

http://www.llamasoftarchive.org/oldsite/gruntingoxfiles/ztoilet.html (Accessed: 18 October 2018).

MinTER, J. (2005a) Neon. Available at:

http://www.minotaurproject.co.uk/neon.php (Accessed: 6 November 2018).

MinTER, J. (2005b) VLM - History. Available at:

http://www.minotaurproject.co.uk/vlm.php (Accessed: 6 November 2018).

Morgana, C. (2010) 'Introduction Artists Re:Thinking Games', in CatLow, R., GARRET, M., and MoRGana, C. (eds) Artists re:Thinking games. Liverpool, UK: Liverpool University Press, pp. 7-14.

MORRIS, J. W. (2015) Selling digital music, formatting culture. Oakland, CA: University of California Press.

MoRRIS, S. (2003) 'WADs, Bots and Mods: Multiplayer FPS Games as Co-creative Media', in DiGRA '03 - Proceedings of the 2003 DiGRA International Conference: Level Up. DiGRA, Utrecht, The Netherlands. Available at: http://www.digra.org/wp-content/uploads/digital-library/05150.21522.pdf.

Moseley, R. (2013) 'Playing games with music (and vice versa):

Ludomusicological perspectives on guitar hero and rock band', in CoOK, N. and PetTengiLl, R. (eds) Taking it to the bridge: Music as performance. Ann Arbor, MI: University of Michigan Press.

Moss, R. (2015) Remembering Nuon, the gaming chip that nearly changed the world -but didn't, Ars Technica. Available at: https://arstechnica.com/gaming/2015/06/ before-the-ps2-nuon-famously-tried-and-failed-to-combine-dvd-and-gameconsoles/ (Accessed: 14 July 2019).

NeWman, J. (2012) Best before: Videogames, supersession and obsolescence. Milton Park, Abingdon: Routledge.

NEWSWIRE, P. (1994) 'JAGUAR CD-ROM UNDER \$200; ATARI REVEALS CD-ROM AND OUTSTANDING CD’, 23 June. 
Nooney, L. (2014) 'Easter Eggs', in Ryan, M.-L., EMERSOn, L., and RoberTson, B. J. (eds) The Johns Hopkins Guide to Digital Media. Baltimore, MD: Johns Hopkins University Press, pp. 165-166.

Patterson, Z. (2009) 'From the Gun Controller to the Mandala: The Cybernetic Cinema of John and James Whitney’, Grey Room, 36, pp. 36-57.

PATTESon, T. (2016) Instruments for New Music: Sound, Technology, and Modernism. Oakland, CA: University of California Press.

PiCKERING, A. (2010) The Cybernetic Brain: Sketches of Another Future. Chicago: University of Chicago Press. Available at:

http://ebookcentral.proquest.com/lib/columbia/detail.action?docID=530446 (Accessed: 30 May 2018).

RetroTech: Atari Video Music - The Migraine Machine (2017). Available at: https:// www.youtube.com/watch?v=wle0eqBwtL8 (Accessed: 17 July 2019).

RHA, J. (1995) VLM codes?? Do they exist?, rec.games.video.atari. Available at: https://groups.google.com/forum/\#!original/rec.games.video.atari/iYfTh00SwJ8/ mTONq2cNDcEJ (Accessed: 30 October 2018).

RugGILL, J. E. (2015) Tempest: Geometries of play. Ann Arbor, MI: University of Michigan Press.

SONY COMPuter ENTERTAinment (2011) PlayStation ${ }^{\circledR}$ Cumulative Production Shipments of Hardware | CORPORATE INFORMATION | Sony Computer Entertainment Inc. Available at: https://web.archive.org/web/20110524023857/http://www.scei.co.jp/corporate/ data/bizdataps_e.html (Accessed: 26 October 2018).

SPIEGEL, L. (1998) 'Graphical GROOVE: Memorial for the VAMPIRE, a visual music system', Organised Sound, 3(3), pp. 187-191. doi:

10.1017/S1355771898003021.

StUART, K. (2005) 'Jeff Minter vs Xbox 360: How Microsoft bought the light synth vision', The Guardian: Technology, 5 July. Available at:

https://www.theguardian.com/technology/gamesblog/2005/jul/05/jeffmintervs (Accessed: 10 November 2018).

Summers, T. (2016) 'Analyzing Video Game Music: Sources, Methods and a Case Study', in KAMP, M., Summers, T., and SwEENEY, M. (eds) Ludomusicology: 
Approaches to video game music. Sheffield, UK: Equinox Publishing (Genre, music and sound).

SuRMan, D. (2010) 'Everyday Hacks: Why Cheating Matters', in CATLOW, R., GARRET, M., and MoRgana, C. (eds) Artists re:Thinking games. Liverpool, UK: Liverpool University Press, pp. 74-77.

WARK, M. (2004) A hacker manifesto. Cambridge, MA: Harvard University Press. WEINEL, J. (2018) Inner sound: Altered states of consciousness in electronic music and audio-visual media. New York, NY: Oxford University Press.

Whyte, R. (2019) 'A Light in Sound, a Sound-like Power in Light': Light and/as Music in the History of the Color Organ. PhD diss. Columbia University.

WiLson, M. and MeLEGA, J. (1981) 'Woody and Steina Vasulka: From Feedback to Paganini', Artlines, May, pp. 8-10. Available at: http://www.vasulka.org/archive/ 4-20a/Artlines(5056).pdf (Accessed: 10 November 2018).

Wolf, M. J. P. (ed.) (2008) The video game explosion: A history from PONG to Playstation and beyond. Westport, Conn: Greenwood Press.

YAK (JEFF MINTER) (1996) Re: VLM Codes, rec.games.video.atari.

YAK (JEFF MinTER) (2005) Neon/360 Controls part 1, YakYak. Available at:

http://www.yakyak.org/viewtopic.php?f=25\&t=46432 (Accessed: 15 July 2019). 